\title{
Composition and antioxidant activity of water-soluble oligosaccharides from Hericium erinaceus
}

\author{
YILING HOU, XIANG DING and WANRU HOU \\ Key Laboratory of Southwest China Wildlife Resources Conservation (Ministry of Education), \\ College of Life Sciences, China West Normal University, Nanchong, Sichuan 637009, P.R. China
}

Received September 23, 2013; Accepted June 26, 2014

DOI: $10.3892 / \mathrm{mmr} .2014 .3121$

\begin{abstract}
Oligosaccharide are carbohydrate molecules, comprising repeating units joined together by glycosidic bonds. In recent years, an increasing number of oligosaccharides have been reported to exhibit various biological activities, including antitumor, immune-stimulation and antioxidation effects. In the present study, crude water-soluble oligosaccharides were extracted from the fruiting bodies of Hericium erinaceus with water and then successively purified by diethylaminoethyl-cellulose 52 and Sephadex G-100 column chromatography, yielding one major oligosaccharide fraction: Hericium erinaceus oligosaccharide (HEO-A). The structural features of HEO-A were investigated by a combination of monosaccharide component analysis by thin layer chromatography, infrared spectroscopy, nuclear magnetic resonance spectroscopy, scanning electron microscopy and high-performance gel permeation chromatography. The results indicated that HEO-A was composed of D-xylose and D-glucose, and the average molecular size was $\sim 1,877 \mathrm{Da}$. The antioxidant activity of HEO-A was evaluated using three biochemical methods to determine the scavenging activity of HEO-A on 1,1-diphenyl-2-picrylhydrazyl, hydrogen peroxide and 2,2'-azino-bis(3-ethylbenzthiazoline-6-sufonic acid) diammonium radicals. The results indicated that HEO-A may serve as an effective healthcare food and source of natural antioxidant compounds.
\end{abstract}

\section{Introduction}

Oligosaccharides are frequently found as components of glycoproteins or glycolipids; therefore, these molecules are often used as chemical markers, particularly for cell recognition. When oligosaccharides are consumed, any undigested

Correspondence to: Dr Xiang Ding, Key Laboratory of Southwest China Wildlife Resources Conservation (Ministry of Education), College of Life Sciences, China West Normal University, 1 Shida Road, Nanchong, Sichuan 637009, P.R. China

E-mail: biostart8083@126.com

Key words: oligosaccharide, antioxidant activity, Hericium erinaceus remnants act as fuel for the intestinal microflora. The effects exerted on the bacterial groups may be either stimulatory or suppressive, depending on the type of oligosaccharide $(1,2)$. Clinical studies have demonstrated that the administration of fructo-oligosaccharides, galacto-oligosaccharides or inulin can enhance the levels of useful intestinal bacteria and simultaneously reduce the number of harmful bacteria $(3,4)$.

The sialyl-Lewis(x) oligosaccharide, identified in a study in 2011 (5), is believed to be the most abundant carbohydrate receptor in the outer coating, or zona pellucida (ZP), of human female ova. This oligosaccharide has been suggested to play a pivotal role in the binding of sperm to the ZP, leading to fertilization. Sialyl-Lewis(x) branches are more abundant on the ova than on any other type of body cell, forming a complete coating. The identification this prominently featuring receptor on the human ova may enhance research into the field of infertility (5).

In numerous organisms, the production of energy for the fueling of biological processes is dependent on oxidation. However, the uncontrolled production of superoxide anion free radicals is involved in the onset of a number of diseases, including cancer, atherosclerosis and degenerative processes associated with aging (6). Thus, it is essential to develop effective and natural antioxidants to protect the human body from free radicals and certain chronic diseases (7).

Hericium erinaceus is a type of fungi belonging to the Hericium family, which grows in Xiaojin county of Sichuan in China at altitudes of $\sim 3,700 \mathrm{~m}$ (8). In the present study, water-soluble oligosaccharides were extracted and purified from the fruiting bodies of Hericium erinaceus using diethylaminoethyl (DEAE)-cellulose and Sephadex G-200 column chromatography. To the best of our knowledge, the present study is the first to characterize the chemical structure of the extracted Hericium erinaceus oligosaccharide (HEO-A). The antioxidant activity of HEO-A was evaluated using three biochemical methods.

\section{Materials and methods}

Chemicals. The fruiting bodies of Hericium erinaceus were collected in Xiaojin county of Sichuan, China, and were authenticated by Professor Zhirong Yang (College of Life Sciences, Sichuan University, Chengdu, China). At the same time, a voucher specimen was preserved in the Key Laboratory of Southwest China Wildlife Resources Conservation 
(Nanchong, China). DEAE-cellulose 52 and Sephadex G-200 were purchased from Sigma-Aldrich (Shanghai, China). Monosaccharide standards and Dextran T-500, T-110, T-70, T-40, and T-10, were purchased from Beijing Biodee Biotechnology Co., Ltd. (Beijing, China). All other reagents used were of analytical grade.

Extraction, purity and fractionation of oligosaccharides from Hericium erinaceus. Once the fruiting bodies (200 g) of Hericium erinaceus were soaked with $95 \%$ ethanol (EtOH), the residue was dried and then extracted with boiling water three times ( $3 \mathrm{~h}$ each). Following the concentration, dialysis and centrifugation of the filtrate, three equivalents of $95 \% \mathrm{EtOH}$ were added to the supernatant to precipitate the crude oligosaccharides (HEO, $21.7 \mathrm{~g}$; recovery, 10.85\%). Following deproteination using the Sevag method (9), LEO (5 g) was purified using a DEAE-cellulose column (Tris- $\mathrm{HCl}, \mathrm{pH} \mathrm{7.0,4.5 \times 50} \mathrm{cm}, \mathrm{Cl}^{-}$) and eluted stepwise with $0,0.1,0.2,0.3,0.4,0.5$ and $1.0 \mathrm{M} \mathrm{NaCl}$. The eluate was monitored by the phenol-sulfuric acid method (10). The $0 \mathrm{M} \mathrm{NaCl}$ eluate was concentrated, lyophilized and purified on a Sephadex G-200 column $(2.6 \times 60 \mathrm{~cm})$. The resulting HEO-A was obtained by the above processes at a yield rate of $0.12 \%(0.24 \mathrm{~g})$ for the starting material.

Assessment of molecular weight. High-performance gel permeation chromatography was performed to measure the molecular weight of HEO-A (11). The column was calibrated with standard T-series Dextran (T-500, T-110, T-70, T-40 and T-10). Data were processed using the Waters Millennium32 Gel Permeation Chromatography software (Waters Corp. Milford, MA, USA).

Monosaccharide composition analysis. The HEO-A $(5.0 \mathrm{mg})$ was hydrolyzed with $2 \mathrm{M}$ trifluoroacetic acid at $110^{\circ} \mathrm{C}$ for $6 \mathrm{~h}$ by acid-catalyzed hydrolysis (12). Excess acid was removed by co-distillation with methyl alcohol following the completion of hydrolysis. The hydrolysate was used for thin layer chromatography (TLC) analysis as previously described (13) using acetoacetate-pyridine-EtOH-water (8:5:1.5:1) as the developing solvent and a diphenylamine-aniline system [85\% phosphoric acid solution $(140 \mathrm{ml})$ containing $8 \mathrm{ml}$ diphenylamine and $8 \mathrm{~g}$ aniline] as the developer system.

Polarimetry(ORD), ultraviolet (UV) and infrared (IR) spectroscopic analysis. HEO-A was assessed using UV spectroscopy at wavelengths of 200-600 nm. IR analysis of the HEO sample was obtained by grinding a mixture of oligosaccharide with dry $\mathrm{KBr}$ and then pressing into a mold. Spectra were run in the $4,000-400 \mathrm{~cm}^{-1}$ range (14). ORD analysis of the HEO sample was obtained using using an HK7_SGW_1 automatic optical polarimeter (Shanghai Jingke Scientific Instrument Co., Ltd., Shanghai, China) at room temperature.

Nuclear magnetic resonance (NMR) experiment. ${ }^{1} \mathrm{H}-\mathrm{NMR}$ and ${ }^{13} \mathrm{C}$-NMR spectra were recorded on a Varian Unity INOVA 400/45 (Varian Medical Systems, Palo Alto, CA, USA) in $\mathrm{D}_{2} \mathrm{O}$ with tetramethylsilane as the internal standard (15).

Assessment of the 1,1-diphenyl-2-picrylhydrazyl (DPPH) free radical scavenging activity of $\mathrm{HEO}-\mathrm{A}$. The $\mathrm{DPPH}^{-}$radical scavenging activity of HEO-A was measured according to the method described by Braca et al (16). The percentage scavenging activity was calculated using the following formula: Scavenging effect $(\%)=(1-A$ sample $/ A$ control $) \times 100$, where $A$ control is the absorbance of the control (DPPH solution without sample) and $A$ sample is the absorbance of the test sample (DPPH solution plus test sample or positive control). Vitamin c ( Vc) and butylated hydroxytoluene (BHT) were used as positive controls.

Assessment of the 2,2'-azino-bis(3-ethylbenzthiazoline-6-sufonic acid) diammonium (ABTS) radical scavenging activity. To determine the $\mathrm{ABTS}^{+}$scavenging activity, the assay was performed as described by Auddy et al (14). ABTS ${ }^{+}$ radicals were produced by reacting ABTS and ammonium persulfate and incubating the mixture at room temperature in the dark for $16 \mathrm{~h}$. A total of $2 \mathrm{ml} \mathrm{HEO-A} \mathrm{solution} \mathrm{at} \mathrm{different}$ concentrations and $2 \mathrm{ml} \mathrm{ABTS}^{+}$radical solution $(0.7 \mathrm{mM})$ were then added. The absorbance was measured immediately at $734 \mathrm{~nm}$. A control reaction was performed without the extract. The percentage scavenging of $\mathrm{ABTS}^{+}$radicals was calculated as follows: Scavenging effect $(\%)=[1-(A$ sample $-A$ sample blank) $/ A$ control] $\times 100$, where $A$ control was the absorbance of the control group in the $\mathrm{ABTS}^{+}$radical generation system, $A$ sample was the absorbance of the test group and $A$ sample blank was the absorbance of the sample only. Vc was used as a positive control.

Assessment of the hydroxyl radical scavenging activity. The ability of HEO-A to scavenge hydrogen peroxide was determined according to the method of Smirnoff and Cumbes (17). The percentage scavenging of hydroxyl radicals was calculated as follows: Scavenging effect $(\%)=[1-(A$ sample $-A$ sample blank) $/ A$ control] $\mathrm{x} 100$, where $A$ control was the absorbance of the control group in the hydroxyl radical generation system, $A$ sample was the absorbance of the test group and $A$ sample blank was the absorbance of the samples only. Vc was used as a positive control.

Statistical analysis. All values are expressed as the mean \pm standard deviation of three replications. Statistical analyses were performed using the Student's t-test and one-way analysis of variance. Values of $\mathrm{P}<0.05$ were considered to indicate a statistically significant difference.

\section{Results and Discussion}

Extraction, purity and composition of oligosaccharides. The crude oligosaccharide was obtained from the fruiting bodies of Hericium erinaceus with a yield of $8.7 \%$. Following fractionation using DEAE-cellulose 52 and Sephadex G-200 column chromatography, $180 \mathrm{mg}$ HEO-A was obtained from the $0 \mathrm{M} \mathrm{NaCl}$ eluate and detected using the phenol-sulfuric acid assay as a single peak (Figs. 1 and 2). The homogeneity of the oligosaccharide was then elucidated. An absence of absorption at 280 and $260 \mathrm{~nm}$ in the UV absorption spectra of HEO-A demonstrated the absence of protein and nucleic acid in this oligosaccharide. In addition, HEO-A exhibited the same optical rotation, $[\alpha]^{20}-11.2^{\circ}$ (concentration, $0.5 \mathrm{~g} / 100 \mathrm{ml}$ water), in different low concentrations (5, 10 and $15 \%)$ of EtOH, 


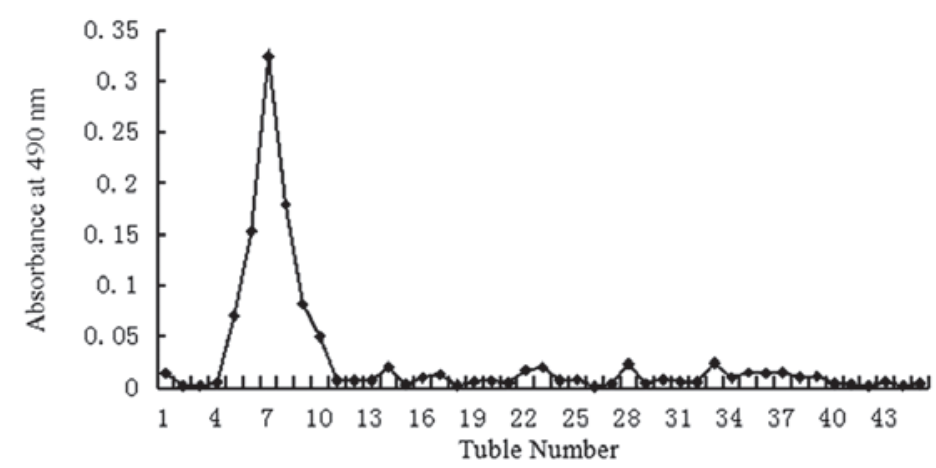

Figure 1. Chromatogram of Hericium erinaceus oligosaccharide on a diethylaminoethyl-cellulose 52 column eluted with distilled water and a stepwise gradient of $\mathrm{NaCl}(0,0.1,0.2,0.3,0.4$ and $0.5 \mathrm{M})$.

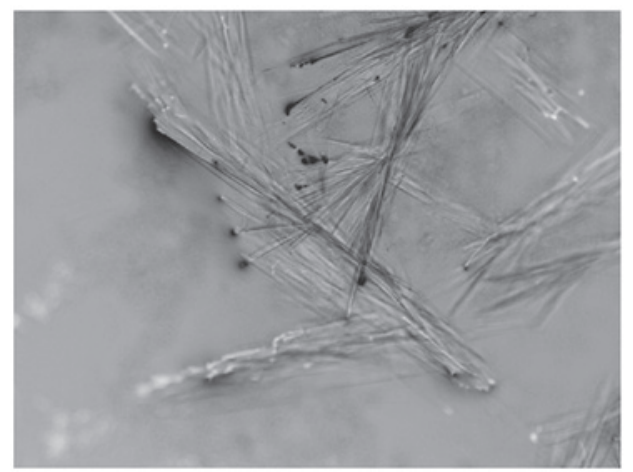

Figure 2. Hericium erinaceus oligosaccharide was visualized by scanning electron microscopy. Magnification, x200.

as shown using an HK7-SGW-1 automatic optical polarimeter at room temperature. The weight-average molecular weight of HEO-A was $\sim 1,877$ Da (Fig. 3). The two monosaccharides, D-glucose (D-Glu) and D-xylose (D-Xyl), were identified by analyzing the hydrolysate of HEO-A using TLC.

Structure elucidation of HEO-A. The intensity of bands around $3,422.38 \mathrm{~cm}^{-1}$ in the IR spectrum (Fig. 4) was due to the hydroxyl stretching vibration of the oligosaccharide and, as expected, they were broad. The bands in the region of $2,922.70 \mathrm{~cm}^{-1}$ were due to a $\mathrm{C}-\mathrm{H}$ stretching vibration and the bands in the region of $1,648.42 \mathrm{~cm}^{-1}$ were due to associated water (18). The strong absorption bands at $1,155.58$ and $1,060.45 \mathrm{~cm}^{-1}$ in the range of $1,200-1,000 \mathrm{~cm}^{-1}$ in the IR spectrum suggested that the monosaccharide in HEO-A had a pyranose ring. The strong absorption bands at $1,402.57 \mathrm{~cm}^{-1}$ were due to the $\mathrm{C}-\mathrm{H}$ bending vibration, and the bands in the region of $923.69-707.59 \mathrm{~cm}^{-1}$ were due to the C-H rocking vibration. In addition, the characteristic absorptions at $707.59 \mathrm{~cm}^{-1}$ indicated $\alpha$-configurations existing in the oligosaccharide, which was consistent with the anomeric proton signals at $\delta 5.06$ and 5.07 in the ${ }^{1} \mathrm{H}-\mathrm{NMR}(400 \mathrm{MHz})$ spectrum (Fig. 5). The signals at $\delta$ 3.0-3.9 were the signal peaks of remaining protons, which were mostly formed by a number of overlapping signal peaks. Among them, the signals at $\delta 3.79,3.78$ and 3.77 were ascribed to the $\alpha-\mathrm{H}$ of the methyl group in xylose and the signals at $\delta 3.55$ corresponded with the $\beta-\mathrm{H}$ of the methyl group in xylose. Signals at $\delta 3.53,3.52$ and 3.45 were the signal peaks of the other hydrogen atoms in xylose. The signals at $\delta 3.65$ and 3.63 were the signal peaks of the hydroxy-methyl group in glucose and the signals at $\delta 3.80$ indicated the hydrogen atom of C-3 of glucose. The hydrogen signal of water was observed at $\delta 4.67$. The ${ }^{1} \mathrm{H}-\mathrm{NMR}$ data were consistent with monosaccharide analysis data. According to previous studies, the resonances in the region of 95-100 ppm in the ${ }^{13} \mathrm{C}$ NMR $(400 \mathrm{MHz})$ spectrum of HEO-A were attributed to the anomeric carbon atoms of D-Glu and D-Xyl (Fig. 6) (19).

Determination of the DPPH radical scavenging activity of HEO-A. Previous studies have shown that oligosaccharides composed of different monosaccharides and with different molecular weights may exhibit different antioxidant activities, particularly in terms of scavenging free radicals (20). As excess free radicals are harmful to human health, the free radical scavenging activity of the extracted oligosaccharide against $\mathrm{DPPH}^{-}$, hydroxyl radicals and superoxide anions was evaluated.

The stable $\mathrm{DPPH}^{-}$radical is commonly used to measure the antioxidant activity of a sample (21). Furthermore, the $\mathrm{DPPH}^{-}$assay is a more time-efficient method than other strategies. In the presence of hydrogen-donating antioxidants, the characteristic purple of an alcoholic solution containing $\mathrm{DPPH}^{-}$radicals and unpaired electrons changes to yellow. This discoloration occurs due to the single paired electrons. Thus, the $\mathrm{DPPH}^{-}$radical scavenging activity of HEO-A was determined by measuring the discoloration and absorbance values at $517 \mathrm{~nm}$, and the results were compared with those of BHT. Fig. 7 shows the scavenging activity of the purified oligosaccharide samples on the $\mathrm{DPPH}^{-}$radical. These results showed that the $\mathrm{IC}_{50}$ value of HEO-A for eliminating $\mathrm{DPPH}^{-}$radicals was $\sim 12.5 \mathrm{mg} / \mathrm{ml}$, which indicated that HEO-A had a notable effect on scavenging $\mathrm{DPPH}^{-}$radicals, particularly when added at high quantities. However, the inhibition ability was lower than that of BHT and Vc.

Assessment of the $\mathrm{ABTS}^{+}$radical cation scavenging activity of HEO-A. The $\mathrm{ABTS}^{+}$radical scavenging activity of HEO-A was measured spectrophotometrically at $734 \mathrm{~nm}$. Upon interaction with the extract at various concentrations, the absorbance of the $\mathrm{ABTS}^{+}$radical cations was decreased dose dependently, and the $\mathrm{IC}_{50}$ value of HEO-A was $0.93 \mathrm{mg} / \mathrm{ml}$ 


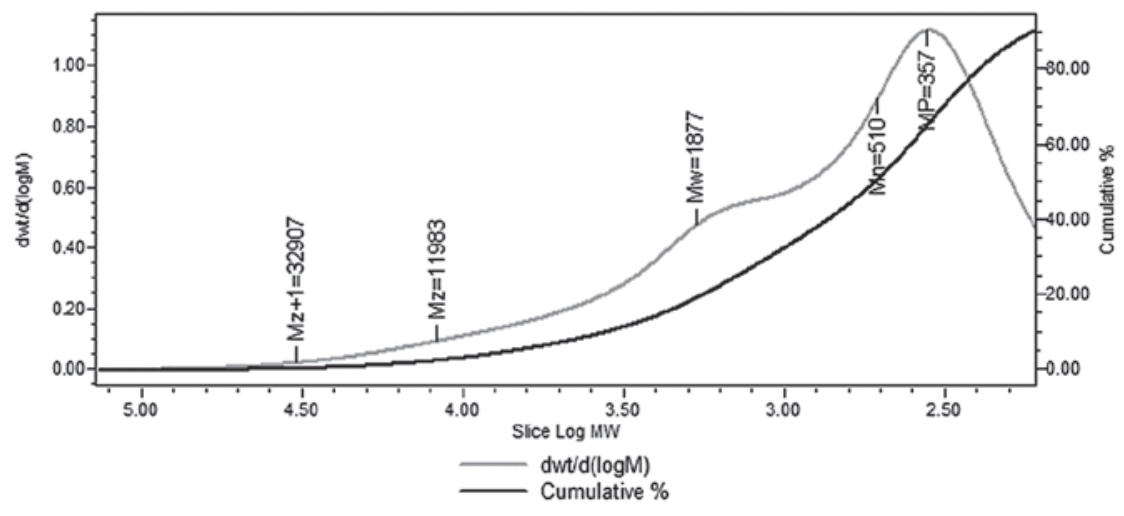

Figure 3. Molecular weight of Hericium erinaceus oligosaccharide.

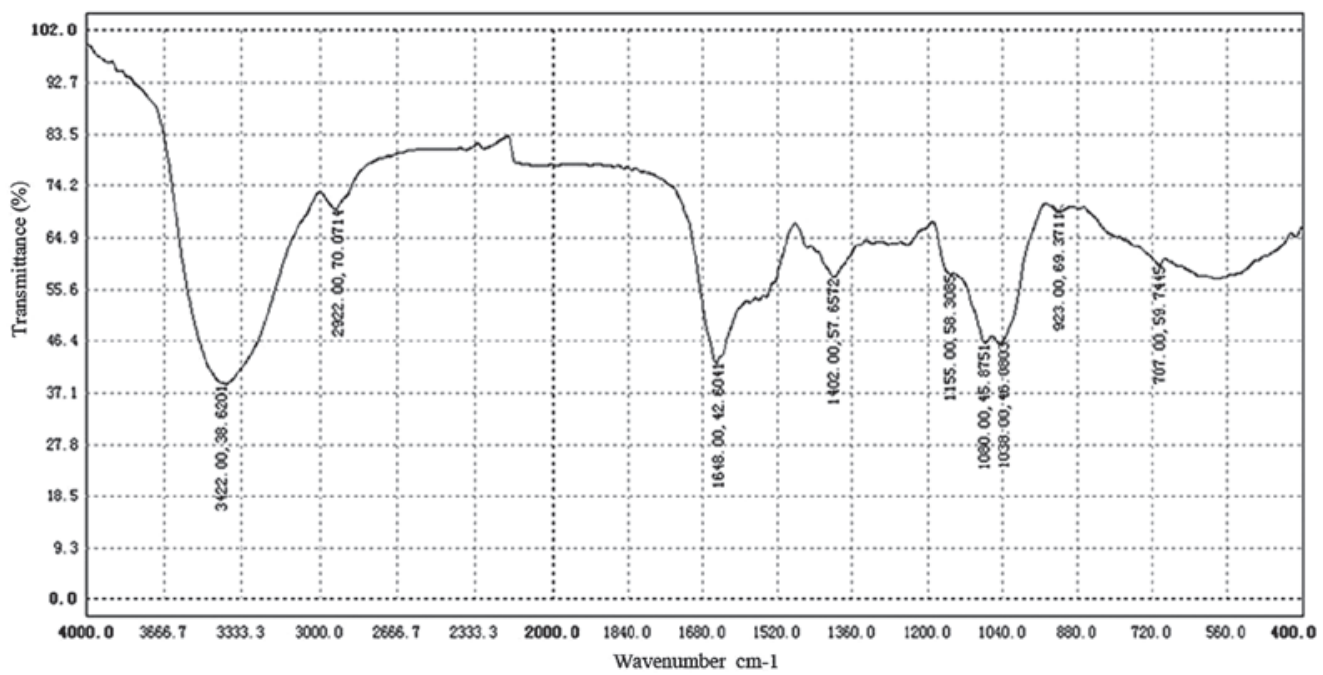

Figure 4. Spectrum of Hericium erinaceus oligosaccharide obtained by Fourier transform infrared spectroscopy.

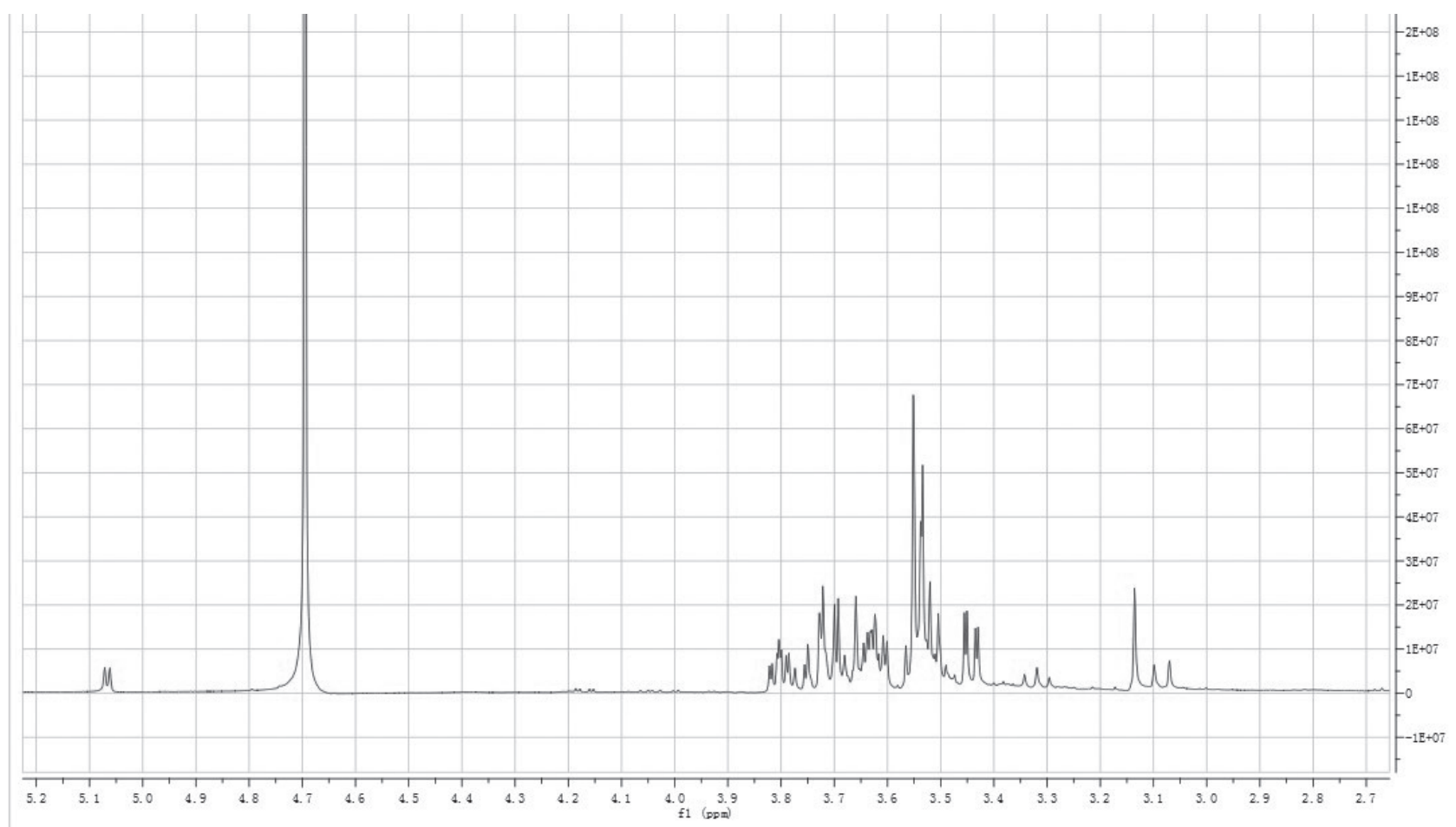

Figure 5. ${ }^{1} \mathrm{H}$-nuclear magnetic resonance spectrum of Hericium erinaceus oligosaccharide. 


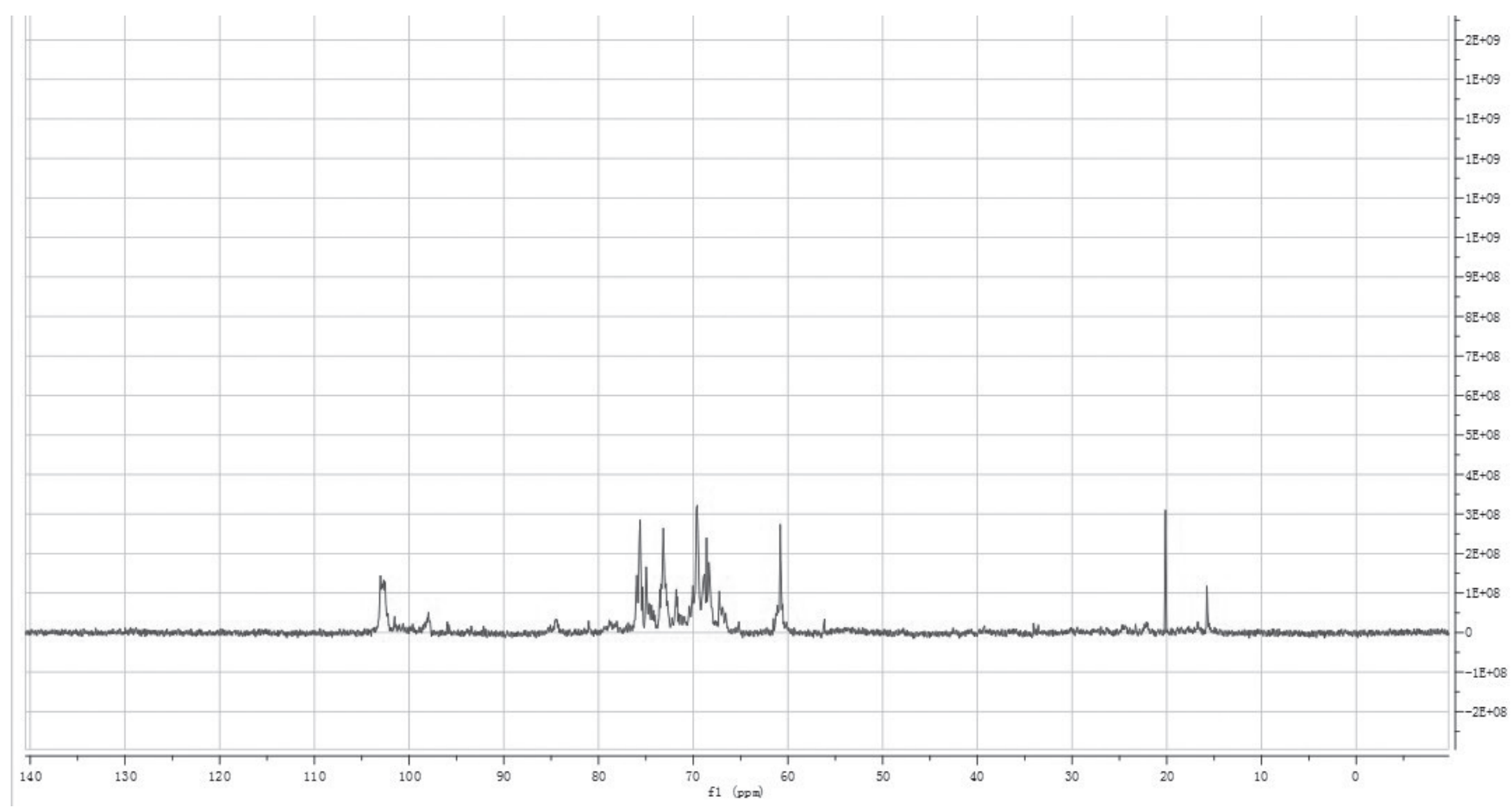

Figure $6 .{ }^{13} \mathrm{C}$-nuclear magnetic resonance spectrum of Hericium erinaceus oligosaccharide.

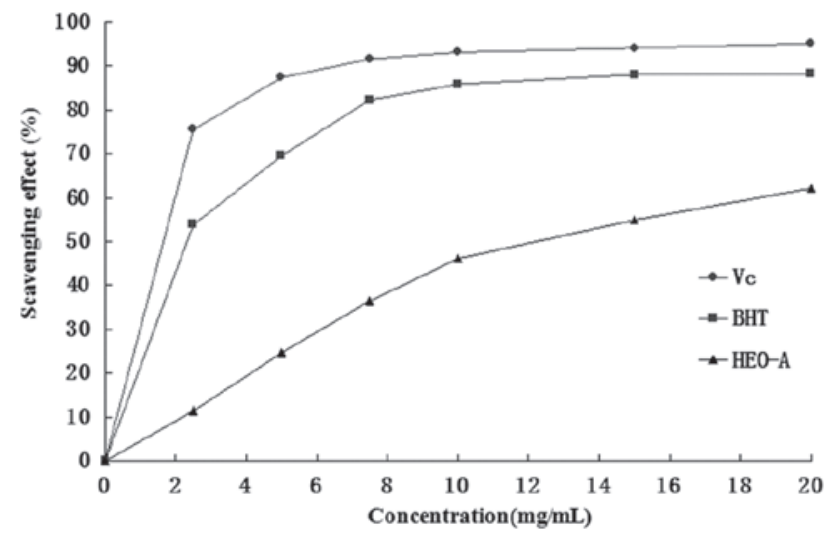

Figure 7. Scavenging effects of HEO-A on 1,1-diphenyl-2-picrylhydrazyl radicals. Vc, vitamin c; BHT, butylated hydroxytoluene; HEO-A, Hericium erinaceus oligosaccharide.

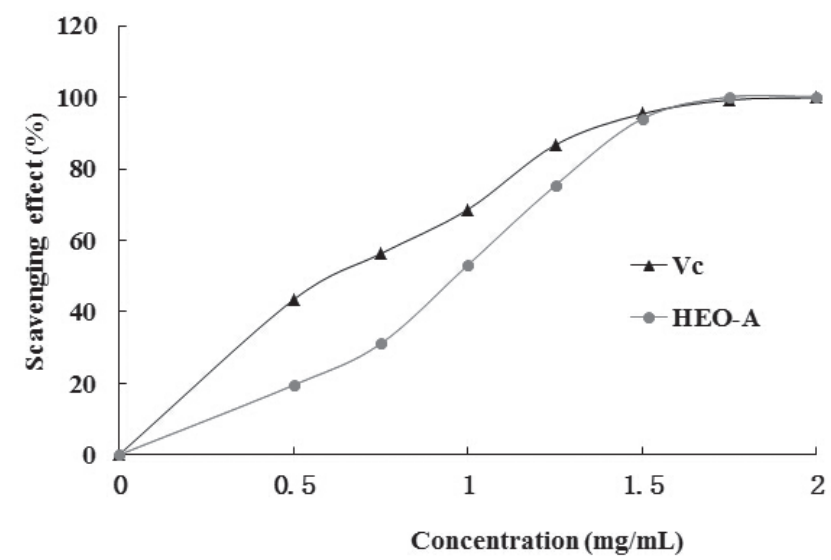

Figure 8. Scavenging effects of HEO-A on 2,2'-azino-bis(3-ethylbenzthiazoline-6-sufonic acid) diammonium radicals. HEO-A, Hericium erinaceus oligosaccharide; Vc, vitamin c.

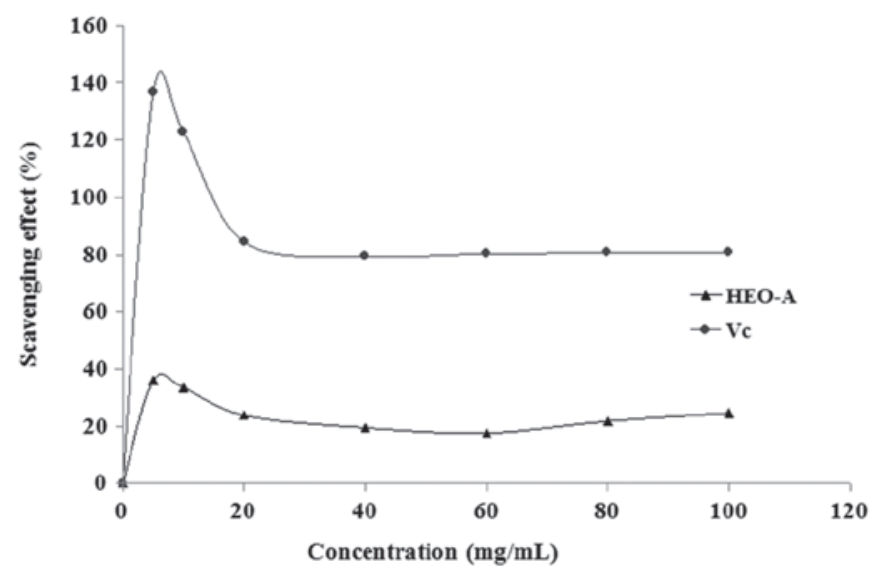

Figure 9. Scavenging effects of HEO-A on hydroxyl radicals. HEO-A, Hericium erinaceus oligosaccharide; $\mathrm{Vc}$, vitamin $\mathrm{c}$.

(Fig. 8). However, the scavenging activity of HEO-A was lower than that of Vc.

Assessment of the hydroxyl radical scavenging activity of $H E O-A$. The hydroxyl radical is one of the most reactive and dangerous reactive oxygen species to human health. The hydroxyl radicals generated through the Fenton reaction in this system were scavenged by HEO-A. Fig. 9 shows the percentage hydroxyl radical scavenging effects of HEO-A at different doses. At the test concentrations, HEO-A exhibited a concentration-dependent scavenging effect on the hydroxyl radicals, which showed that the purified oligosaccharide exhibited weaker hydroxyl radical-scavenging effects than $\mathrm{Vc}$ at the same dose.

Conclusion. According to the results above, Hericium erinaceus may be introduced as a possible valuable source of 
oligosaccharides, which exhibits unique antioxidant properties.

\section{Acknowledgements}

This study was supported by the National Natural Science Foundation of China (grant nos. 31200012 and 31400016), the Application Foundation Project of Sichuan Province (grant no. 2013JY0094), the Science and Technology Support Project of Sichuan Province (grant nos. 2014SZ0020 and 2014FZ0024), The Cultivate Major Projects of Sichuan Province (grant no. 14CZ0016), and the Doctor Startup Foundation Project of China West Normal University (grant nos. $11 \mathrm{~B} 019$ and 11B020).

\section{References}

1. Bode L: Human milk oligosaccharides: prebiotics and beyond. Nutr Rev 67 (Suppl 2): S183-S191, 2009.

2. De Filippo C, Cavalieri D, Di Paola M, Ramazzotti M, Poullet JB, Massart S, Collini S, Pieraccini G and Lionetti P: Impact of diet in shaping gut microbiota revealed by a comparative study in children from Europe and rural Africa. Proc Natl Acad Sci USA 107: 14691-14696, 2010.

3. Macfarlane GT, Steed H and Macfarlane S: Bacterial metabolism and health-related effects of galacto-oligosaccharides and other prebiotics. J Appl Microbiol 104: 305-344, 2008.

4. Bertozzi CR and Kiessling LL: Chemical glycobioloy. Science 291: 2357-2364, 2001.

5. Pang PC, Chiu PC, Lee CL, et al: Human sperm binding is mediated by the sialyl-Lewis(x) oligosaccharide on the zona pellucida. Science 333: 1761-1764, 2011.

6. Mau JL, Tsai SY, Tseng YH and Huang SJ: Antioxidant properties of hot water extracts from Ganoderma tsugae Murrill LWT - Food Sci Technol 38: 589-597, 2005.

7. Kinsella JE, Frankel EN, German JB and Kanner J: Possible mechanisms for the protective role of antioxidants in wine and plant foods. Food Technol 47: 85-89, 1993.

8. Fan WW and Huang HH: Advances on Hericium erinaceus Polysaccharides. Food science 29: 355-358, 2008.
9. Staub AM: Removal of protein - Sevag method. In: Methods in Carbohydrate Chemistry Volume 5: General Polysaccharides. Whistler RL (ed). Academic Press, New York, NY, pp 5-6, 1965.

10. Dubois M, Gillis KA, Hamilton JK, Rebers PA and Smith F: Colorimetric method for determination of sugars and related substances. Anal Chem 28: 350-356, 1956.

11. Yamamoto $Y$, Nunome $T$, Yamauchi $R$, Kato $K$ and Sone Y: Structure of an exocelluluar polysaccharide of Lactobacillus helveticus TN-4, a spontaneous mutant strain of Lactobacillus helveticus TY1-2. Carbohydr Res 275: 319-332, 1995.

12. Yu RM, Yin Y, Yang W, Ma WL, Yang L, Chen XJ, Zhang Z, Ye B and Song LY: Structural elucidation and biological activity of a novel polysaccharide by alkaline extraction from cultured Cordyceps militaris. Carbohydr Polym 75: 166-171, 2009.

13. Partridge SM: Aniline hydrogen phthalate as spraying reagent for chromatography of sugars. Nature 164: 443, 1949.

14. Auddy B, Ferreira M, Blasina F, Lafon L, Arredondo F, Dajas F, Tripathi PC, Seal T and Mukherjee B:Screening of antioxidant activity of three Indian medicinal plants, traditionally used for the management of neurodegenerative diseases. J Ethnopharmacol 84: 131-138, 2003.

15. Cao W, Li XQ, Liu L, Wang M, Fan HT, Li C, et al: Structural analysis of water-soluble glucans from the root of Angelica sinensis (Oliv.) Diels. Carbohydr Res 341: 1870-1877, 2006.

16. Braca A, De Tommasi N, Di Bari L, Pizza C, Politi M and Morelli I: Antioxidant principles from Bauhinia terapotensis. J Nat Prod 64: 892-895, 2001.

17. Smirnoff N and Cumbes QJ: Hydroxyl radical scavenging activity of compatible solutes. Phytochemistry 28: 1057-1060, 1989.

18. Barker SA, Bourne EJ, Stacey M and Whiffen DH: Infra-red spectra of carbohydrates. Part I. Some derivatives of D-glucopyranose. J Chem Soc 1954: 171-176, 1954.

19. Wang ZJ, Luo DH and Liang ZY: Structure of polysaccharides from the fruiting body of Hericium erinaceus Pers. Carbohydr Poly 57: 241-247, 2004.

20. Chen Y, Xie MY, Nie SP, Li C and Wang YX: Purification, composition analysis and antioxidant activity of a polysaccharide from the fruiting bodies of Ganoderma atrum. Food Chem 107: 231-241, 2008.

21. Ye H, Wang KQ, Zhou CH, Liu J and Zeng XX: Purification, antitumor and antioxidant activities in vitro of polysaccharides from the brown seaweed Sargassum pallidum. Food Chem 111: 428-432, 2008 